1 Fundação Oswaldo Cruz (Fiocruz), Instituto Aggeu Magalhães - Recife (PE), Brasil.

Orcid: https://orcid org/0000-0001-51555522

daianny.ps@hotmail.com

2 Fundação Oswaldo Cruz (Fiocruz), Instituto Aggeu Magalhães - Recife (PE),

Brasil.

Orcid: https://orcid

org/0000-0001-8299-

2901

iracema_alves@hotmail.com

3 Fundação Oswaldo Cruz (Fiocruz), Instituto Aggeu Magalhães - Recife (PE),

Brasil.

Orcid: https://orcid

org/0000-0002-9620-

2621

george@cpqam.fiocruz.br

4 Fundação Oswaldo Cruz (Fiocruz), Instituto Aggeu Magalhães - Recife (PE),

Brasil.

Orcid: https://orcid. org/0000-0001- 6592-

0762

anabrito1009@gmail.com

${ }^{5}$ Fundação Oswaldo Cruz (Fiocruz), Instituto Aggeu Magalhães - Recife (PE),

Brasil.

Orcid: https://orcid.

org/0000-0002-5290-

092X

naideteodosio@gmail.com

\section{Histórico de violência entre mulheres que fazem uso de crack no estado de Pernambuco, Brasil}

\author{
History of violence among women who use crack in the state of \\ Pernambuco, Brazil
}

Daianny de Paula Santos', Iracema de Jesus Almeida Alves Jacques², George Tadeu Nunes Diniz ${ }^{3}$, Ana Maria de Brito ${ }^{\mathbf{4}}$, Naíde Teodósio Valois Santos $\mathbf{5}$

DOI: $10.1590 / 0103-1104201811906$

RESUMO Neste artigo, abordou-se o histórico de violência contra mulheres em contexto de uso abusivo de crack. Trata-se de um estudo transversal, envolvendo 243 usuárias de crack atendidas pelo Programa Atitude, em Pernambuco, entre 2014 e 2015. Observou-se que a maioria era jovem, negra, com baixa escolaridade e renda, início precoce da vida sexual, em uso compulsivo de crack, morando na rua e comercializando o corpo como a principal fonte de renda. A maioria (96,2\%) relatou histórico de violência: psicológica (83,5\%), física (87,7\%) e sexual (55,1\%), praticadas por parentes/amigos. Fatores como desigualdades sociais e de gênero foram relevantes nas situações de violência observadas.

PALAVRAS-CHAVE Violência contra a mulher. Drogas ilícitas. Vulnerabilidade em saúde.

ABSTRACT In this article, it was discussed the history of violence against women in the context of abusive use of crack. It is a cross-sectional study, involving 243 crack users attended by the Atitude Program, in Pernambuco, between 2014 and 2015. It was observed that they were mostly young, black, with low schooling and low-income, early start of sexual life, in compulsive use of crack, living on the street and commercializing the body as the main source of income. The majority (96.2\%) reported a violence history: psychological (83.5\%), physical (87.7\%) and sexual (55.1\%), practiced by relatives/friends. Factors such as social and gender inequalities were relevant in the situations of violence observed.

KEYWORDS Violence against women. Street drugs. Health vulnerability. 


\section{Introdução}

A violência é uma questão sociocultural complexa, de grande magnitude e impactos sociais, reconhecida como um relevante problema de saúde pública, podendo ser perpetrada em múltiplos contextos, principalmente nos cenários de injustiças e discriminações. Segundo a Organização Mundial da Saúde (OMS), compreende-se por violência o uso intencional da força física ou do poder entre indivíduos e comunidades, que ocasione ou tenha possibilidade de causar lesão, desenvolvimento prejudicado e morte ${ }^{1,2}$.

No campo relativo a álcool e outras drogas, a relação entre a violência e o uso abusivo de substâncias psicoativas é algo difícil de ser mensurado, pois os contextos violentos não são resultantes apenas das condutas individuais, mas possuem interface com a estrutura social de desigualdades e injustiças que afeta indivíduos e comunidades, cujos direitos sociais são negados ou minimizados pelo Estado. Por outro lado, as políticas proibicionistas, alinhadas com a chamada 'guerra às drogas', criminalizam o uso de drogas, principalmente o crack, estigmatizando, legitimando e naturalizando práticas de violência contra as pessoas que consomem drogas ${ }^{\mathbf{3}, \mathbf{4}}$.

Segundo o Anuário Brasileiro de Segurança Pública, o Brasil lidera no cenário mundial números absolutos de homicídios por ano, de modo que, ainda de acordo com esses dados, são mais de 56 mil mortes violentas, sendo estimado que $50 \%$ delas estejam associadas à repressão ao uso de drogas. Apesar de o perfil carcerário e de uso do crack ser predominantemente masculino, na última década, houve um crescimento de $567,4 \%$ da presença da população feminina em presídios, estando a maioria dessas prisões relacionada ao envolvimento coadjuvante das mulheres no tráfico de drogas, principalmente no mercado do crack e seus derivados ${ }^{4,5}$.

A relação entre drogas e violência torna-se ainda mais complexa quando se observam as situações envolvendo relações de gênero e contextos de vulnerabilidade social, em que estão inseridas as mulheres que fazem uso de crack. Essas diferentes interfaces têm resultado em maior frequência e gravidade das agressões no cotidiano dessas mulheres, sendo a violência perpetrada pelas pessoas mais próximas à sua dinâmica de vida, tais como cônjuges, parentes, amigos/colegas de tráfico, clientes, traficantes, polícia, entre outros ${ }^{5,6}$.

A Pesquisa Nacional sobre o Uso de Crack no Brasil também trouxe outros aspectos importantes sobre vulnerabilidade à violência envolvendo questões de gênero. $\mathrm{O}$ estudo revelou que $46,6 \%$ das mulheres que consomem crack em lugares públicos referiram história de violência sexual, enquanto, entre os homens, esse percentual foi seis vezes menor (7,5\%). A Pesquisa chama, ainda, atenção para a maior proporção de comercialização do sexo entre as mulheres (29,9\%), ou seja, 23 vezes maior quando comparada com os homens (1,3\%), o que também implica uma maior exposição a agressões, bem como reflete que a trajetória de vida é amplamente desfavorável às mulheres ${ }^{7}$.

Compreendendo as diversas condições que fragilizam e expõem a mulher usuária de crack a situações de violência, o presente estudo objetiva analisar o histórico de violência dessa população, bem como ampliar conhecimentos acerca de seu perfil sociodemográfico, vida sexual e uso de drogas.

\section{Material e métodos}

Estudo transversal de caráter descritivo que utilizou o banco de dados de Pesquisa Sociocomportamental ${ }^{\mathbf{8}}$ aprovada pelo Comitê de Ética em Pesquisa em seres humanos, sob o parecer CAE 25250413.6.0000.5190. A referida pesquisa é um inquérito epidemiológico realizado com uma amostra probabilística de usuários de crack atendidos em um Programa de Atenção Integral aos Usuários de Drogas e Seus Familiares, da Secretaria Executiva de Políticas sobre Drogas de Pernambuco, que tem por objetivo responder a situações de risco pessoal e social 
em decorrência do uso abusivo de drogas, sem o caráter de tratamento contra a dependência.

A referida pesquisa incluiu 1.062 usuários de crack, sendo 819 do sexo masculino e 243 do sexo feminino, com idade maior ou igual a 18 anos, que consumiram crack por mais de 25 dias nos últimos seis meses. Os dados foram coletados a partir da realização de questionário sociocomportamental, no período de agosto de 2014 a agosto de 2015. Para o presente estudo, foram selecionadas todas as mulheres, considerando aquelas que sofreram algum tipo de violência ou não. $\mathrm{Na}$ análise, não foram incluídas as mulheres transexuais e travestis. Contudo, foram inseridas as nove pessoas que referiram ter nascido do sexo feminino e apresentando identidade de gênero diferente do sexo biológico. Foram levantadas variáveis relacionadas ao histórico de violência de acordo com a natureza da agressão (psicológica, física e sexual), principais agressores, faixa etária da vítima no momento da agressão e periodicidade da violência. Além disso, investigaram-se os aspectos sociodemográficos, a vida sexual e o padrão de uso de crack.

Considerou-se por histórico de violência o relato de agressões psicológicas e/ou físicas e/ou sexuais sofridas em qualquer momento da vida dessas mulheres. Para identificar a ocorrência de qualquer violência, era necessária resposta afirmativa de pelo menos uma dessas questões: (1) Alguma vez na vida você já foi ameaçada, humilhada, chantageada, perseguida, ridicularizada ou impedida de ver algum familiar?; (2) Alguém já te esbofeteou, deu murros, espancou, queimou ou tentou enforcar você?; (3) Alguém já te feriu com uma faca, estilete, caco de vidro, revólver ou outro objeto?; (4) Alguém já forçou você, contra a sua vontade, a ter relações sexuais?.

As entrevistas ocorreram em um ambiente protegido, sem o uso de coerção institucional ou policial. As participantes foram informadas sobre os objetivos, procedimentos, riscos, benefícios e caráter voluntário da pesquisa, sendo garantido o anonimato e a confidencialidade das informações prestadas.

Utilizou-se o software Statistical Package for the Social Scienses (SPSS), versão 20, para obter as medidas de tendência central para as variáveis quantitativas contínuas e frequências absolutas e relativas para variáveis categóricas, sendo os resultados apresentados em gráficos e tabelas. $\mathrm{Na}$ análise das características das agressões sofridas pelas entrevistadas, as diferenças entre as proporções foram verificadas por meio do teste qui-quadrado de proporção, adotando-se um nível de significância estatística de 5\%.

\section{Resultados e discussão}

O consumo de crack, que até a década de 1990 era observado, principalmente, na população jovem masculina, recentemente, vem sendo identificado como um fenômeno crescente entre as mulheres, atrelado a grande vulnerabilidade social e desigualdade de gênero, que, entre outros aspectos, envolve a comercialização do corpo para conseguir a droga e a exposição a situações de violência ${ }^{5}$.

Com relação às características sociodemográficas (tabela 1), a maioria das entrevistadas tinha entre 18 e 34 anos (85,6\%), negras (66,3\% pardas e $18,1 \%$ pretas), solteiras (60,5\%), com filhos (83,9\%), e pequena proporção ingressou no ensino médio (13,5\%). No tocante à moradia, $52,7 \%$ estavam em situação de rua nos 30 dias anteriores à entrevista, sendo esse resultado maior do que o observado por Bastos e Bertoni ${ }^{9}$ nas cenas abertas de uso de crack no Brasil (45,6\%). 
Tabela 1. Características sociodemográficas e relacionadas à vida sexual de mulheres que fazem uso de crack. Programa Atitude, Pernambuco, 2014/2015

\begin{tabular}{|c|c|c|}
\hline \multirow{2}{*}{ VARIÁVEIS } & \multicolumn{2}{|c|}{$(N=243)$} \\
\hline & $\mathrm{n}$ & $\%$ \\
\hline \multicolumn{3}{|l|}{ Faixa etária (anos) } \\
\hline 18 a 24 & 98 & 40,3 \\
\hline 25 a 34 & 110 & 45,3 \\
\hline 35 a 44 & 31 & 12,8 \\
\hline 45 a 54 & 4 & 1,6 \\
\hline \multicolumn{3}{|l|}{ Raça/Cor } \\
\hline Parda & 161 & 66,3 \\
\hline Preta & 44 & 18,1 \\
\hline Branca & 34 & 14,0 \\
\hline Amarela & 4 & 1,6 \\
\hline \multicolumn{3}{|l|}{ Situação Conjugal } \\
\hline Solteira & 147 & 60,5 \\
\hline Casada ou em união estável & 72 & 29,6 \\
\hline Separada, divorciada e viúva. & 24 & 8,2 \\
\hline \multicolumn{3}{|l|}{ Número de Filhos } \\
\hline Não tem & 39 & 16,0 \\
\hline Com filhos & 204 & 83,9 \\
\hline \multicolumn{3}{|l|}{ Escolaridade } \\
\hline Ensino superior incompleto & 02 & 0,8 \\
\hline Ensino Médio completo & 20 & 8,2 \\
\hline Ensino Médio incompleto & 11 & 4,5 \\
\hline Ensino Fund. II completo & 18 & 7,4 \\
\hline Ensino Fund. II incompleto & 69 & 28,4 \\
\hline Ensino Fund. I completo & 61 & 25,1 \\
\hline Ensino fund. Incompleto & 42 & 17,3 \\
\hline Alfabetização & 19 & 7,8 \\
\hline Nunca estudou & 01 & 0,4 \\
\hline \multicolumn{3}{|l|}{ Estuda atualmente } \\
\hline Sim & 12 & 4,9 \\
\hline Não & 231 & 95,1 \\
\hline \multicolumn{3}{|c|}{ Lugar onde viveu a maior parte do tempo nos últimos 30 dias } \\
\hline Rua & 128 & 52,7 \\
\hline Programa Atitude ou abrigo & 12 & 5,3 \\
\hline Casa própria ou familiar & 54 & 22,6 \\
\hline Casa alugada ou amigos, ou quarto de hotel/pensão & 48 & 19,8 \\
\hline Prisão/delegacia & 01 & 0,4 \\
\hline
\end{tabular}


Tabela 1. (cont.)

\begin{tabular}{lrr}
\hline Mora com alguém que usa crack & & \\
\hline Sim & 86 & 51,5 \\
Não & 81 & 48,5 \\
\hline Trabalha atualmente & 128 & 52,7 \\
\hline Sim & 115 & 47,3 \\
Não & 54 & 47,8 \\
\hline Motivo de não estar trabalhando & 22 & 19,5 \\
\hline Doença/tratamento para dependência química & 6 & 5,3 \\
Procura, mas sem sucesso & & \\
Uso de drogas & 141 & 58,0 \\
\hline Renda mensal individual & 102 & 42,0 \\
\hline$<1$ salário mínimo & & 5 \\
$\geq 1$ salário mínimo & & \\
\hline
\end{tabular}

Fontes de renda nos últimos 30 dias ${ }^{\star \star}$

Comercialização de sexo (profissional do sexo/troca de sexo por dinheiro)

Família/parceiros/amigos

$95 \quad 39,1$

Trabalho esporádico/bicos

Atividade ilícita que não o tráfico de drogas (ex. furto, roubo)

Esmolas

Participação na venda ou distribuição de drogas (tráfico)

Trabalho por conta própria

Trabalho regular (com ou sem carteira)

Início da vida sexual

Menos de 10 anos

19

10 a 14 anos

15 a 18 anos

Mais de 18 anos

07

Número de parceiros(as) sexuais nos últimos 12 meses

1

37

6 a 10

Fonte: Elaboração própria.

*Os valores de referência do salário mínimo para os anos de 2014 e 2015 foram $R \$ 724,00$ e R $\$ 788,00$, respectivamente; **A diferença entre o total das entrevistadas e o total da variável deve-se à possibilidade de múltiplas respostas. 
A literatura aponta que muitas mulheres que fazem uso de drogas e estão em situação de rua possuem uma história de vida marcada por violência doméstica, envolvendo parentes e parceiros. Elas enxergam as ruas como uma alternativa para se protegerem, ou seja, um escape para a violência que sofrem no seu contexto familiar. Entretanto, ao estarem nas ruas, são novamente vulneradas pela violência, com outros moldes de agressão e perpetradores. Nesse contexto, são frequentes as agressões decorrentes da coerção policial e os conflitos entre os próprios moradores de rua, estranhos e usuários de crack, bem como o envolvimento dessas mulheres com pessoas ou redes que delas se utilizam para exploração sexual ou para o tráfico de drogas ${ }^{9-14}$.

No que se refere a trabalho e renda, foi observado que uma pequena proporção estava inserida no mercado formal de trabalho (4,6\%), sendo a dependência química (47,8\%) apontada pelas usuárias como o central motivo de não estarem trabalhando. A principal fonte de renda foi a comercialização do sexo (39,1\%), aspecto também verificado em estudos com populações usuárias de crack de outras capitais brasileiras ${ }^{11,15,16}$. De modo geral, essa realidade reflete fatores de vulnerabilidade social como a baixa escolaridade e a falta de qualificação profissional, consequências da dificuldade de acesso a melhores condições de ensino e trabalho, num cenário de uso nocivo de crack.

O início precoce da vida sexual, antes dos 15 anos (76,1\%), e a multiplicidade de parceiros, 10 ou mais nos últimos 12 meses (53,1\%), foram frequentes entre as entrevistadas, resultado semelhante ao observado entre mulheres usuárias de crack em São Paulo11,15 e no Canadá12. Tais proporções estão acima das verificadas para a população feminina brasileira ${ }^{\mathbf{1 7}}(\mathbf{1 7 , 0 \%}$ e $10,9 \%$, respectivamente). Tais achados apontam uma maior exposição das mulheres que fazem uso de crack a práticas sexuais desprotegidas e a vitimização por violência sexual, assim como a comercialização do sexo como meio de subsistência e de aquisição de drogas.

O cenário observado neste estudo revela uma outra questão gritante, porém, velada, que é o fato de que a iniciação sexual tão precoce apresentada por essas mulheres representa mais uma violência, que, de acordo com o Artigo 218 do Código Penal Brasileiro, intitula-se 'estupro de vulnerável', ou seja, um fenômeno complexo e multicausal que consiste no constrangimento e/ou na indução de um menor de 14 (catorze) anos de idade à prática de conjunção carnal (penetração pênis-vagina) e/ou a satisfação de quaisquer atos libidinosos. Essa realidade também foi constatada em um estudo realizado com usuárias de crack na cidade de São Paulo ${ }^{15}$, segundo o qual 13 das 75 mulheres entrevistadas foram estupradas ainda virgens, na infância ou pré-adolescência. Dessa forma, a violência tem um peso ainda maior, pois se mostra como um fator determinante nas histórias de vida dessas mulheres, influenciando suas práticas sexuais e constituindo um fator de risco para o abuso e a dependência de drogas na idade adulta.

A raiz dessa problemática, bem como as demais situações de vulnerabilidade dessas mulheres à violência fundamentam-se na estrutura patriarcal regente desde as primeiras sociedades até a atualidade. $\mathrm{O}$ patriarcado consiste em um sistema sociocultural arbitrário de poder de homens sobre mulheres, ou seja, é um regime de domínio masculino no tocante ao corpo, à sexualidade e ao trabalho das mulheres, contribuindo para as concepções sociais de subordinação, diferenciação salarial e personificação do corpo feminino como objeto sexual5,6,15,18,19.

As características relacionadas ao consumo de drogas são descritas na tabela 2. Observa-se que mais de um terço iniciou o uso de crack ainda na adolescência, sendo $14,1 \%$ entre 10 e 15 anos, e $24,9 \%$ entre 16 e 18 anos, com uso diário (75,7\%) e descontrolado (79,4\%). As mulheres possuem perfil de consumo mais precoce e intenso quando comparado com o dos homens ${ }^{20}$. Essas características também foram observadas no estudo nacional sobre o uso de $\mathrm{crack}^{9}$ entre jovens em situação de rua nas cidades 
de São Paulo e Porto Alegre ${ }^{\mathbf{1 4}}$, bem como entre usuários de crack em Nova York ${ }^{21}$. Tais diferenças no padrão de uso de crack entre homens e mulheres apontam para a necessidade de uma melhor compreensão da relação de gênero na cultura de uso de drogas, bem como sobre os fatores sociais imbricados nessa questão.

Tabela 2. Características relacionadas ao consumo de crack por mulheres. Programa Atitude, Pernambuco, 2014/2015

\begin{tabular}{|c|c|c|}
\hline \multirow{2}{*}{ VARIÁVEIS } & \multicolumn{2}{|c|}{$(\mathrm{N}=243)$} \\
\hline & $\mathrm{n}$ & $\%$ \\
\hline \multicolumn{3}{|l|}{ Idade de início do uso de crack } \\
\hline 10 a 15 anos & 34 & 14,1 \\
\hline 16 a 18 anos & 60 & 24,9 \\
\hline 19 a 25 anos & 80 & 33,2 \\
\hline 26 a 39 anos & 63 & 26,1 \\
\hline 40 a 54 anos & 4 & 1,7 \\
\hline \multicolumn{3}{|l|}{ Principal motivo que levou a usar crack } \\
\hline Influência/pressão de amigos & 96 & 39,5 \\
\hline Curiosidade & 58 & 23,9 \\
\hline Problemas familiares & 38 & 15,6 \\
\hline Perdas afetivas/decepções amorosas & 33 & 13,6 \\
\hline Vida ruim/sem perspectivas & 7 & 2,9 \\
\hline Outros & 18 & 4,5 \\
\hline \multicolumn{3}{|l|}{ Com quem costuma usar crack ${ }^{\star}$} \\
\hline Sozinha & 134 & 55,1 \\
\hline Colegas/amigos & 108 & 44,4 \\
\hline Parceiros sexuais & 83 & 34,1 \\
\hline Parentes & 10 & 4,1 \\
\hline Outros & 1 & 0,6 \\
\hline \multicolumn{3}{|c|}{ Frequência semanal de uso de crack antes de ingressar no Programa Atitude } \\
\hline Todos os dias & 184 & 75,7 \\
\hline De 04 a 06 dias & 17 & 7,0 \\
\hline Até 03 dias & 37 & 15,2 \\
\hline Menos de 1 dia & 5 & 2,1 \\
\hline \multicolumn{3}{|c|}{ Autoavaliação da forma de consumir crack antes de ingressar no Programa Atitude } \\
\hline Descontrolada/ compulsivamente & 193 & 79,4 \\
\hline Controlada & 50 & 20,6 \\
\hline \multicolumn{3}{|l|}{ Locais de obtenção do crack } \\
\hline Boca de fumo & 190 & 78,2 \\
\hline Rua & 35 & 14,4 \\
\hline Entrega no domicílio & 14 & 5,8 \\
\hline Outros & 4 & 1,6 \\
\hline
\end{tabular}


Tabela 2. (cont.)

\begin{tabular}{lcr}
\hline Meios de obter crack sem envolver dinheiro* & & \\
\hline Trocar por sexo & 99 & 40,7 \\
Trocar por objetos & 60 & 24,7 \\
Participar de alguma forma do tráfico de drogas & 48 & 19,8 \\
Consertar/limpar coisas, ajudar a fazer um trabalho legal & 18 & 7,4 \\
Participar de trabalho ilegal (roubo etc.) & 18 & 7,4 \\
Trocar por outra droga & 18 & 7,4 \\
Outro & 20 & 8,2 \\
\hline Desejo de realizar tratamento para a dependência & 221 & 97,1 \\
\hline Sim & 7 & 2,9 \\
Não & & 7 \\
\hline
\end{tabular}

Fonte: Elaboração própria.

${ }^{\star}$ A diferença entre o total das entrevistadas e o total da variável deve-se à possibilidade de múltiplas respostas.

No tocante à narrativa de violência, chama atenção que quase a totalidade das mulheres entrevistadas referiu ter vivenciado situações de violência (96,2\%), revelando uma história de vida marcada por agressões psicológicas, físicas e sexuais, mesmo antes de iniciarem o uso de drogas, algumas ainda na infância ou na adolescência. Observa-se, no gráfico 1, que as agressões mais frequentes foram as psicológicas (83,5\%), físicas por espancamento (82,3\%), seguidas pela violência sexual $(55,1 \%)$ e por agressões por meio de arma branca ou de fogo (54,7\%). Comparando com outras populações femininas, as agressões físicas analisadas neste estudo foram superiores àquelas observadas com usuárias de crack paulistas $^{11}$ e com mulheres vítimas de violência atendidas em emergências do Sistema Único de Saúde (SUS) no território nacional22.

Gráfico 1. Tipo de violência sofrida por mulheres que fazem uso de crack, segundo a relação da agressão com o uso de drogas. Programa Atitude, Pernambuco, 2014/2015

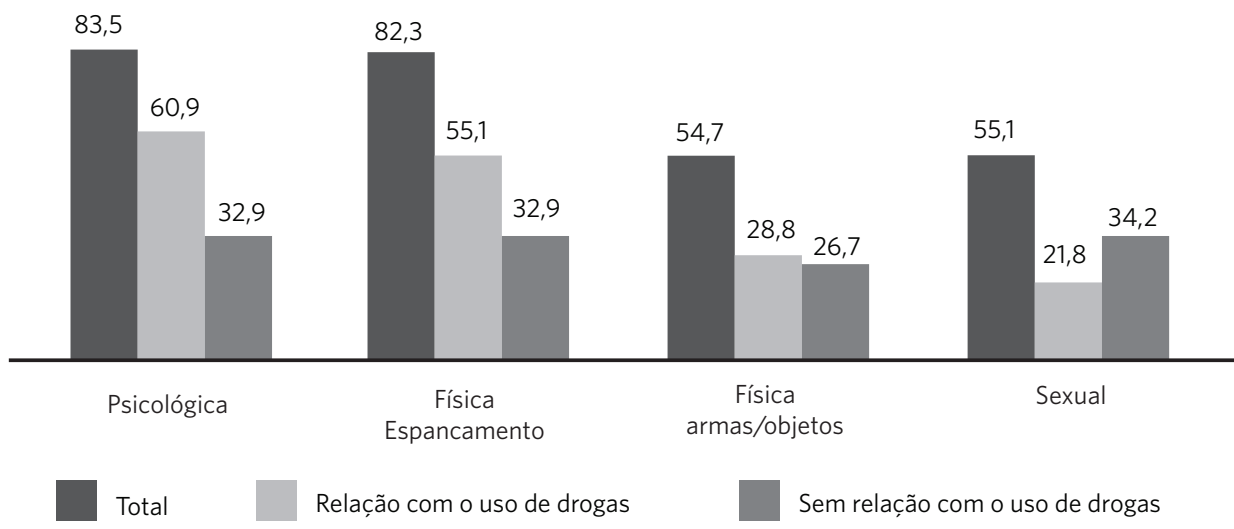

Fonte: Elaboração própria.

Notas: A relação da agressão com o uso de drogas foi estabelecida pelas entrevistadas; Violência psicológica: ameaça, humilhação, chantagem, perseguição, ridicularização, impedimento de ver filho e/ou parente; Violência física por espancamento: relato de ter sido esbofeteada, murros, tapas, queimaduras, entre outras; Violência física por objeto: ferimento por arma de fogo, branca e/ou outro objeto pérfurocortante. 
A presença marcante da violência nessa população também foi verificada em estudos sobre mulheres usuárias de drogas em diferentes cidades brasileiras ${ }^{3,8,15,16,23}$ e no Canadá12; e sobre adolescentes em situação de rua na cidade de São Paulo ${ }^{14}$. Entre as participantes deste estudo, foi observado, também, que o contexto do uso abusivo de drogas mostrou-se um fator relevante sobre as situações de violência vivenciadas.

Ainda no gráfico 1, observa-se o tipo de violência e sua relação com o uso de drogas, de acordo com a percepção das entrevistadas. As agressões relacionadas com o uso de drogas sobressaíram-se no histórico de violência psicológica e física por espancamento, sendo referidas por $60,9 \%$ e $55,1 \%$ das mulheres, respectivamente, para cada tipo de violência. Já no que diz respeito à violência por armas ou envolvendo o uso de outro objeto, não se observa diferença expressiva.

Diferentemente do observado para os demais tipos de agressões, a proporção de usuárias que referiu ter sofrido violência sexual sem relacioná-la com o uso de drogas foi superior à que referiu essa relação. No entanto, é importante ressaltar que a relação entre uso de drogas e violência é muitas vezes mistificada, na medida em que o consumo de substâncias psicoativas é erroneamente situado como fator disparador para as situações de violência, não levando-se em consideração os fatores sociais, culturais e políticos imbricados ${ }^{4,13}$.

Na violência contra a mulher, estudos destacam que, mesmo em contextos em que há uso abusivo de drogas por agressores e/ou vítimas, a raiz da violência tem maior peso por razão da estrutura sociocultural de dominação e exploração que incide sobre a vida das mulheres, sendo expressa pelo machismo e acentuada pela exclusão social 16,18,19,24. Investigações sobre violência entre mulheres que fazem uso de crack demonstram que os atos violentos estão subjugados à estruturação do tráfico de drogas, às relações interpessoais entre usuários e traficantes, bem como às injustiças sociais, que são fatores marcantes nessa população $0^{3,5,8,15,16,23}$.

As características das agressões sofridas pelas mulheres que fazem uso de crack são apresentadas na tabela 3. Destaca-se que, em todos os tipos de violência, os principais agressores foram as pessoas mais próximas as vítimas, tais como familiares, amigos e companheiros. Diferentemente das demais agressões, na violência sexual, constatou-se um maior envolvimento de pessoas estranhas (46,7\%) como autoras do crime.

Tabela 3. Agressor, faixa etária de ocorrência e frequência de agressões sofridas por mulheres que fazem uso de crack, segundo natureza da violência. Programa Atitude, Pernambuco, 2014-2015

\begin{tabular}{|c|c|c|c|c|c|c|c|c|}
\hline \multirow{3}{*}{ VARIÁVEIS } & \multicolumn{8}{|c|}{ Tipo/natureza da agressão } \\
\hline & \multicolumn{2}{|c|}{$\begin{array}{l}\text { Psicológica }{ }^{1} \\
(\mathrm{~N}=203)\end{array}$} & \multicolumn{2}{|c|}{$\begin{array}{c}\text { Física por } \\
\text { espancamento } 2 \\
(\mathrm{~N}=200)\end{array}$} & \multicolumn{2}{|c|}{$\begin{array}{l}\text { Física por objeto } 3 \\
\qquad(\mathrm{~N}=133)\end{array}$} & \multicolumn{2}{|c|}{$\begin{array}{l}\text { Sexual } \\
(N=134)\end{array}$} \\
\hline & $\mathbf{n}$ & $\%$ & n & $\%$ & $\mathbf{n}$ & $\%$ & $\mathrm{n}$ & $\%$ \\
\hline \multicolumn{9}{|l|}{ Agressor 4} \\
\hline $\begin{array}{l}\text { (Ex)companheiro, amigos, conhe- } \\
\text { cidos, familiares }\end{array}$ & 173 & 84,8 & 170 & 84,1 & 105 & 78,3 & 76 & 56,3 \\
\hline Estranho & 36 & 17,7 & 31 & 15,4 & 29 & 21,6 & 63 & 46,7 \\
\hline Traficante & 73 & 35,8 & 42 & 20,8 & 17 & 12,7 & 3 & 2,2 \\
\hline Polícia & 27 & 13,2 & 20 & 9,9 & 7 & 5,2 & 3 & 2,2 \\
\hline p-valor 5 & \multicolumn{2}{|c|}{$<0,0001$} & \multicolumn{2}{|c|}{$<0,0001$} & \multicolumn{2}{|c|}{$<0,0001$} & \multicolumn{2}{|c|}{$<0,0001$} \\
\hline
\end{tabular}


Tabela 3. (cont.)

\begin{tabular}{|c|c|c|c|c|c|c|c|c|}
\hline \multicolumn{9}{|c|}{ Faixa etária da ocorrência da agressão ${ }^{4}$} \\
\hline Menos de 10 anos & 6 & 2,9 & 3 & 1,5 & 2 & 1,5 & 10 & 7,4 \\
\hline 10 a 19 anos & 63 & 30,9 & 57 & 28,2 & 43 & 32,1 & 51 & 37,8 \\
\hline 20 a 24 anos & 64 & 31,4 & 64 & 31,7 & 47 & 35,1 & 46 & 34,1 \\
\hline 25 a 59 anos & 95 & 46,6 & 90 & 44,6 & 50 & 37,3 & 34 & 25,2 \\
\hline p-valor ${ }^{5}$ & \multicolumn{2}{|c|}{$<0,0001$} & \multicolumn{2}{|c|}{$<0,0001$} & \multicolumn{2}{|c|}{$<0,0001$} & \multicolumn{2}{|c|}{$<0,0001$} \\
\hline \multicolumn{9}{|c|}{ Frequência das agressões } \\
\hline Raramente & 95 & 46,6 & 99 & 49,0 & 95 & 70,9 & 101 & 74,8 \\
\hline Às vezes & 49 & 24,0 & 57 & 28,2 & 28 & 20,9 & 21 & 15,6 \\
\hline Frequentemente & 60 & 29,4 & 46 & 22,8 & 11 & 8,2 & 13 & 9,6 \\
\hline$p$-valor 5 & \multicolumn{2}{|c|}{$<0,0001$} & \multicolumn{2}{|c|}{$<0,0001$} & \multicolumn{2}{|c|}{$<0,0001$} & \multicolumn{2}{|c|}{$<0,0001$} \\
\hline
\end{tabular}

Fonte: Elaboração própria.

1Violência psicológica: ameaça, humilhação, chantagem, perseguição, ridicularização, impedimento de ver filho e/ou parente. ${ }^{2}$ Violência física por espancamento: relato de ter sido esbofeteada, murros, tapas, queimaduras, entre outras. ${ }^{\vee}$ Violência física por objeto: ferimento por arma de fogo, branca e/ou outro objeto pérfurocortante. ${ }^{4} \mathrm{~A}$ diferença entre o total de mulheres que sofreu violência e o total da variável deve-se à possibilidade de múltiplas respostas. ${ }^{5} \mathrm{As}$ diferenças entre proporções foram verificadas por meio do teste qui-quadrado de proporção, considerando o nível de significância de 5\%.

A proximidade vítima-agressor observada neste estudo está alinhada à própria caracterização da violência contra a mulher, que, em sua maioria, expressa-se nos espaços privados e representa para as mulheres um maior risco à vida, devido à recidiva das agressões e ao silenciamento das vítimas em razão das relações de poder presentes nesses processos ${ }^{6,18}$. Nas situações em que os agressores foram pessoas que faziam parte do contexto de uso de drogas ilícitas, tais como traficantes e policiais, os cenários violentos estão relacionados ao micropoder presente nos cenários de mercado ilícito e coerção ao uso de crack, o que se dá em espaços públicos como ruas e bocas de fumo, conferindo, assim, uma maior vulnerabilidade a essas mulheres que representam papel coadjuvante no tráfico de drogas e/ou em outras atividades ilícitas.

Entre as mulheres que sofreram algum tipo de violência, verifica-se que as agressões ocorreram desde a infância, sendo mais frequentes a partir da pré-adolescência (10 anos ou mais) e aumentando conforme a idade, com exceção da violência sexual, que diminui. Chama atenção que, na infância dessas mulheres, a agressão mais frequente foi a sexual (7,4\%), com proporção pelo menos duas vezes maior que as demais agressões relatadas nessa faixa etária (menos de 10 anos).

Apesar de não terem sido tão frequentes quando comparadas as outras violências, as agressões sexuais foram referidas por metade das mulheres, chamando a atenção para a precocidade dos abusos, que aconteceram principalmente durante a infância e a adolescência. Fertig$^{16}$ e Aguiar ${ }^{23}$ afirmam que abusos sexuais e físicos ocorridos na infância e na adolescência favorecem danos psicológicos, como baixa autoestima, depressão e ansiedade, bem como influenciam a experimentação de substâncias psicoativas, constituindo um fator de risco para o início do uso de drogas ilícitas, sobretudo do crack. Ademais, a violência sofrida no período infanto-juvenil, além de ocasionar impactos ao desenvolvimento psicossocial, traz a reflexão sobre a gravidade desse assunto e da associação destas três condições que permeiam a história de vida dessas mulheres: estupro, drogas e exploração sexual4,10. 
A proporção das mulheres entrevistadas que sofreram violência sexual alguma vez na vida foi cinco vezes superior à estimada para a população feminina nas capitais brasileiras; e duas vezes maior que a de mulheres em cenas abertas de uso de crack no Brasil, ou seja, $96,3 \%$ e $46,6 \%$, respectivamente ${ }^{9,17}$.

Além disso, algumas características da violência sexual se diferenciaram quando comparadas com os demais tipos de agressões. Importante parcela de mulheres vítimas desse tipo específico de violência (34,2\%) não referiu existir uma relação entre a agressão sexual e o uso de drogas. Esse dado pode estar imbricado com outros fatores além do consumo de drogas, como as desigualdades de gênero e a ideologia sociocultural de apropriação e exploração do corpo da mulher. A partir das correntes teóricas do feminismo e das concepções de gênero, pode-se afirmar que o regime patriarcal, presente de forma milenar na sociedade, é uma estrutura hierárquica que confere aos homens o direito de dominar as mulheres, independentemente da figura humana, e, dessa forma, as mulheres são concebidas enquanto objeto da satisfação sexual masculina e reduzidas socialmente a reprodutoras e prestadoras de serviços sexuais.

Nesse processo, é importante destacar que o gênero, raça/etnicidade e as classes sociais constituem-se como eixos estruturantes da violência contra a mulher, em que, dependendo das condições históricas vivenciadas, um desses fatores poderá sobressair sobre os outros, no entanto, os demais continuam presentes e podem produzir/reproduzir a violência. Ademais, as questões de gênero, raça/cor e pobreza são marcadores sociais que influenciam o grau de vulnerabilidade das mulheres à violência, principalmente daquelas inseridas em cenários de uso abusivo de álcool e outras drogas e de desigualdades sociais ${ }^{6,16,18,20,23}$.

No tocante à periodicidade da violência, observa-se que, em quase todos os tipos, as mulheres referiram que as agressões aconteciam raramente e/ou esporadicamente, fato que causa certo estranhamento quando se leva em consideração o grau de vulnerabilidade social dessa população, bem como o elevado percentual que afirmou ter sido violentado em algum momento da vida. Diferem-se apenas desses achados as agressões psicológica $(29,4 \%)$ e física por espancamento (28,2\%), sendo essas as mais presentes em seu cotidiano.

É possível, também, que esse dado mascare outras características dessa realidade, de que tais mulheres não identificam a violência de seu cotidiano, pois ela se apresenta de forma simbólica e rotineira. $\mathrm{Na}$ maioria das vezes, a violência é naturalizada pelas vítimas, que apenas a reconhecem quando há dano físico grave.

A tabela 4 apresenta as múltiplas comparações entre as agressões de acordo com o tipo de violência. De um total de 243 indivíduos da amostra, foram considerados os sujeitos que tinham respostas (sim ou não) para os quatro tipos de violência. Dessa forma, a análise foi realizada com um total de 240 indivíduos, dos quais, 09 responderam negativamente para as violências. 
Tabela 4. Análise das múltiplas comparações entre agressões segundo o tipo de violência sofrida por mulheres que fazem uso de crack. Programa Atitude, Pernambuco, 2014-2015

\begin{tabular}{|c|c|c|c|c|c|c|}
\hline \multirow{3}{*}{ Tipo de Violência } & \multicolumn{2}{|c|}{ Física Arma ${ }^{3}$} & \multicolumn{2}{|c|}{ Física Espancamento } & \multicolumn{2}{|c|}{ Sexual } \\
\hline & Não & Sim & Não & Sim & Não & Sim \\
\hline & $N(\%)^{4}$ & $\mathrm{~N}(\%)^{4}$ & $\mathrm{~N}(\%)^{4}$ & $N(\%)^{4}$ & $N(\%)^{4}$ & $N(\%)^{4}$ \\
\hline \multicolumn{7}{|l|}{ Psicológica ${ }^{1}$} \\
\hline Não & $23(9,6)$ & $14(5,8)$ & $13(5,4)$ & $24(10,0)$ & $25(10,4)$ & $12(5,0)$ \\
\hline Sim & $84(35,0)$ & $119(49,6)$ & $27(11,2)$ & $176(73,3)$ & $81(33,8)$ & $122(50,8)$ \\
\hline$p$-valor 5 & & 0,0580 & & 0,0031 & & 0,0055 \\
\hline \multicolumn{7}{|l|}{ Sexual } \\
\hline Não & $56(23,3)$ & $50(20,8)$ & $25(10,4)$ & $81(33,8)$ & & \\
\hline Sim & $51(21,2)$ & $83(34,6)$ & $15(6,2)$ & $119(49,6)$ & & \\
\hline p-valor 5 & & 0,0668 & & 0,0316 & & \\
\hline \multicolumn{7}{|c|}{ Física - Espancamento² } \\
\hline Não & $28(11,7)$ & $12(5,0)$ & & & & \\
\hline Sim & $79(32,9)$ & $121(50,4)$ & & & & \\
\hline$p$-valor 5 & & 0,0012 & & & & \\
\hline
\end{tabular}

Fonte: Elaboração própria.

1Violência psicológica: ameaça, humilhação, chantagem, perseguição, ridicularização, impedimento de ver filho e/ou parente. ${ }^{2}$ Violência física por espancamento: relato de ter sido esbofeteada, murros, tapas, queimaduras, entre outras. ${ }^{2}$ Violência física por objeto: ferimento por arma de fogo, branca e/ou outro objeto pérfurocortante. ${ }^{4} \mathrm{~A}$ diferença entre o total de mulheres que sofreu violência e o total da variável deve-se à possibilidade de múltiplas respostas. ${ }^{5}$ As diferenças entre proporções foram verificadas por meio do teste qui-quadrado de proporção, considerando o nível de significância de 5\%.

Para as mulheres que foram vítimas de múltiplas agressões, observou-se uma predominância de 176 (73,3\%) para a violência psicológica, acompanhada da violência física por espancamento, ao mesmo tempo $(\mathrm{p}=0,0031)$. Também se observou que, das $121(50,4 \%)$ mulheres que sofreram violência por espancamento, houve o envolvimento da violência por ameaça por arma e outros objetos $(p=0,0012)$, bem como verificou-se comportamento parecido para a violência sexual, com o envolvimento da violência física por espancamento para 119 (49,6\%), ou com o envolvimento da violência psicológica para $122(50,8 \%)$.

Esses achados revelam o caráter de multiplicidades entre as violências e os graus de lesões sofridas pelas vítimas, bem como a utilização de naturezas distintas de agressão enquanto instrumentos de controle no momento do ato violento.

\section{Considerações finais}

Os resultados encontrados reforçam a gravidade da situação dessa população e provocam o debate sobre a estruturação e a ampliação do SUS, por meio de suas redes de atenção, para oferta de acolhimento e seguimento dessa demanda.

Os achados também apontam para necessidades que transcendem as ofertas que, tradicionalmente, são de responsabilidade do campo da saúde, pois também envolvem a atuação de outros setores da sociedade, como educação, trabalho, renda e justiça. Essa temática não se esgota neste 
debate, tendo em vista que investigações sobre violência-gênero-drogas dependem de instrumentos e teorias capazes de fazer uma aproximação do universo de significados sobre violência, tentando responder a questões muito particulares, com um nível de realidade que não pode ser, na maioria das vezes, quantificado.

Logo, é de extrema necessidade a continuidade de outros estudos sobre violência e gênero por meio de análises qualitativas que entendam de forma mais aprofundada como as condições de vida, o uso abusivo de drogas e as relações de gênero determinam e/ou condicionam situações de violência, e de que forma todos esses fatores incidem sobre a vida dessas mulheres.

\section{Colaboradores}

Santos DP contribuiu substancialmente para a concepção, análise e interpretação dos dados. Jacques IJAA contribuiu significativamente para a revisão crítica do conteúdo. Diniz GTN contribuiu significativamente para a revisão crítica do conteúdo. Brito AM participou da aprovação final do manuscrito. Santos NTV contribuiu substancialmente para a concepção, análise e interpretação dos dados.

\section{Referências}

1. Krug EG, Dahlberg LL, Mercy JA, et al. World report on violence and health [internet]. Geneva: WHO; 2002 [acesso em 2016 jul 12]. Disponível em: http:// www.who.int/violence_injury_prevention/violence/ world_report/en/introduction.pdf.

2. Minayo MCS. Violência e Saúde. Rio de Janeiro: Fiocruz; 2006.

3. Lopes EPA, Sapori LF, Medeiros R. Crack: Um desafio Social. Cad. Saúde Pública. 2012 fev-abr; 2(28):405406.

4. Fiori M, Tófoli LF. Uso de drogas, danos e violência. Dossiê Descriminalização STF: Argumentos pela Cri- minalização e Evidências Científicas. Plataforma Bras Pol Drogas. 2015 [acesso em 2016 abr 18]; 2:2-4. Disponível em: http://pbpd.org.br/publicacao/dossie-descriminalizacao-das-drogas-e-o-stf/.

5. Lins J. O uso de crack entre mulheres tendências e desafios. Rev Cultura UFPE. 2011 dez; 28:237-250.

6. Cisne M. Direitos humanos e violência contra mulheres: uma luta contra a sociedade patriarcal-racista-capitalista. Rev Serviço Social. 2015; 18(1):138-154.

7. Bastos FI, Bertoni N. Perfil dos usuários de crack e/ ou similares no Brasil. Livreto Epidemiológico. Secretaria Nacional de Políticas sobre Drogas. 2013 [aces- 
so em 2016 maio 26]; 1:25-184. Disponível em: http:// portal.fiocruz.br/pt-br/content/maior-pesquisa-sobrecrack-j\%C3\%Al-feita-no-mundo-mostra-o-perfil-do-consumo-no-brasil.

8. Santos NTV. Vulnerabilidade e prevalência de HIV e sífilis em usuários de drogas no Recife: resultados de um estudo respondent-driven sampling [tese]. Recife: Fundação Oswaldo Cruz; 2013. 184 p.

9. Bastos FI, Bertoni N. Perfil dos usuários de crack e/ ou similares no Brasil. Livreto Epidemiológico. Secretaria Nacional de Políticas sobre Drogas. 2013 [acesso em 2016 maio 26]; 1:25-184. Disponível em: http:// portal.fiocruz.br/pt-br/content/maior-pesquisa-sobrecrack-j\%C3\%Al-feita-no-mundo-mostra-o-perfil-do-consumo-no-brasil.

10. Coid J, Petruckevitch A, Feder G, et al. Relation between childhood sexualand physical abuse and risk of revictimisation in women: across-sectional survey. Lancet. 2001; 358:450-454.

11. Oliveira LG, Nappo SA. Caracterização da cultura de crack na cidade de São Paulo: padrão de uso controlado. Rev Saúde Pública. 2008; 42:664-671.

12. Bugay V, Johnson JL, Varcoe C, et al. Women's health and use of crack cocaine in context: Structural and 'everyday’ violence. Intern J Drug Policy. 2010; 1:321-329.

13. Chaves TV, Sanchez MS, Ribeiro LA, et al. Fissura por crack: comportamentos e estratégias de controle de usuários e ex-usuários. Rev Saúde Pública. 2011; 45:1168-1175.

14. Raup LM, Adorno RC. Jovens em situação de rua e usos de crack: um estudo etnográfico em duas cidades. Rev. Bras. Adolescência e Conflitualidade. 2011; 4:52-67.

15. Nappo AS, Sanchez ZVM, Oliveira LG, et al. Comportamento de risco de mulheres usuárias de crack em relação às DST/AIDS. Centro Brasileiro de Informações sobre Drogas - CEBRID. Livreto Epidemiológico. 2004 [acesso em 2016 jan 10]; 1:31-93.
Disponível em: http://abramd.org/wp-content/uploads/2014/06/Usuarias-de-crack-CEBRID.pdf.

16. Aguiar CMD. Vivências sexuais de mulheres jovens usuárias de crack [dissertação]. Recife: Universidade Federal de Pernambuco; 2014. 106 p.

17. Brasil. Ministério da Saúde. PCAP: Pesquisa de Conhecimentos, Atitudes e Práticas na População Brasileira. Brasília, DF: MS; 2011.

18. Saffioti HI. Gênero, Patriarcado e Violência. São Paulo: Expressão Popular; 2004.

19. Moraes MA, Silva RE. Mulheres, Crack e Contextos de Vulnerabilidades. In: Lage AC, Dantas CC, Bernardes DAM, et al. Dossiê sobre drogas. Estudos Universitários. Recife: UFPE; 2011. p. 251-267.

20. Santos NTV. Vulnerabilidade e prevalência de HIV e sífilis em usuários de drogas no Recife: resultados de um estudo respondent-driven sampling [tese]. Recife: Fundação Oswaldo Cruz; 2013. 184 p.

21. Koester S, Schwartz J. Crack, Gangs, Sex, and Powerlessness: A View from Denver, In: Ratner SR. Crack Pipe as Pimpe. An Ethnographic Investigation of Sex-for-Crack Exchanges. New York: Lexitngton Books; 1992. p. 187-203.

22. Mascarenhas MDM, Silva MMA, Malta DC, et al. Perfil epidemiológico dos atendimentos de emergência por violência no Sistema de Serviços Sentinelas de Vigilância de Violências e Acidentes (Viva) - Brasil, 2006. Rev Epidemiol Serv Saúde. 2009; 8(1):17-18.

23. Fertig A. Histórias de vida de usuárias de crack [tese]. Porto Alegre: UFRS; 2013. 154 p.

24. Franconi F, Campesi I, Occhioni S, et al. Sex and gender in adverse drug events, addiction, and placebo. Hand Exp Pharmacol. 2012; 214(1):107-126

Recebido em 26/11/2017

Aprovado em 30/10/2018

Conflito de interesses: inexistente

Suporte financeiro: Secretaria de Vigilância em Saúde do

Ministério da Saúde do Brasil (Edital 20/2013) 